\title{
SLAMFI Promotes Methotrexate Resistance via Activating Autophagy in Choriocarcinoma Cells
}

This article was published in the following Dove Press journal:

Cancer Management and Research

\section{Dazun Shi \\ Yu Zhang \\ Yan Tian}

Department of Gynecology and Obstetrics, Xiangya Hospital, Central South University, Changsha, Hunan Province, People's Republic of China
Correspondence: Yan Tian Department of Gynecology and Obstetrics, Xiangya Hospital, Central South University, Changsha 410008, People's Republic of China Email tianyxycsu@I63.com
Objective: The acquisition of chemoresistance to methotrexate (MTX) still remains one of the major challenges for choriocarcinoma treatment. Herein, we aimed to evaluate the potential role of Signaling Lymphocytic Activation Molecule Family Member 1 (SLAMF1) as a possible regulator of chemoresistance to MTX in choriocarcinoma.

Material and Methods: MTX-resistant JEG3 and JAR sublines (JEG3/MTX, JAR/MTX) were used to study SLAMF1 function. CCK8 assay and soft agar assay were conducted to measure the cell viability and clonogenesis of choriocarcinoma cells, respectively; MDC incorporation assay was conducted for the quantification of intracellular autophagy; BrdU labeling was used to assess the proliferative potential of choriocarcinoma cells; SLAMF1 protein expression was analyzed by Western blotting.

Results: Upregulation of SLAMF1 expression was observed in MTX-resistant JEG3/MTX and JAR/MTX sublines compared to their parental JEG3 and JAR cell lines, respectively. Knockdown of SLAMF1 markedly attenuated cell viability and soft agar clonogenesis after incubation with MTX in JEG3/MTX and JAR/MTX cells. In contrast, constitutive expression of SLAMF1 rescued cell survival soft agar clonogenesis in JEG3 and JAR cells treated with MTX. Moreover, autophagy is apparently activated in MTX-resistant JEG3/MTX and JAR/MTX sublines compared to their parental cell lines. Autophagy inhibitor 3-methyladenine and bafilomycin A1 enhanced MTX-induced cytotoxicity in MTX-resistant JEG3 and JAR sublines. Further, SLAMF1 might activate autophagy-related mechanism to promote resistance to MTX in choriocarcinoma cells. Depletion of SLAMF1 suppressed autophagy and induced apoptosis in MTX-treated JEG3/MTX and JAR/MTX cells.

Conclusion: SLAMF1 might promote MTX resistance via activating protective autophagy in choriocarcinoma cell lines. Targeting SLAMF1 might be a useful therapeutic strategy to sensitize choriocarcinoma cells to MTX-based regimens.

Keywords: choriocarcinoma, methotrexate, drug resistance, SLAMF1, autophagy

\section{Introduction}

Choriocarcinoma is a very rare malignancy occurred in the placenta of pregnant women. The main biochemical marker, used in treatment and prognosis for choriocarcinoma is human chorionic gonadotropin (hCG). Choriocarcinoma is often characterized by its fast-growing and aggressive nature, which ultimately leads to patient death. ${ }^{1}$ Methotrexate (MTX) has been successfully used for the treatment of choriocarcinoma for decades. ${ }^{2}$ However, chemoresistance to MTX still represents a major challenge for choriocarcinoma treatment. A considerable fraction $(20 \sim 30 \%)$ of low-risk choriocarcinoma may fail to achieve complete remission after MTX treatment. ${ }^{3,4}$ Moreover, about 10 20\% 
patients with high-risk choriocarcinoma may render an incomplete remission to MTX-containing regimens. ${ }^{5,6}$ Although chemoresistance to MTX in choriocarcinoma has been well recognized, its regulatory mechanisms are not fully understood. A number of molecular mechanisms responsible for MTX resistance are documented, including drug efflux via ATP-binding-cassette (ABC) transporters, ${ }^{7,8}$ upregulation of dihydrofolate reductase (DHFR) gene, ${ }^{9}$ inhibition of apoptosis, ${ }^{10}$ involvement of interferon signaling, ${ }^{11}$ and enrichment of cancer stem cell fraction. ${ }^{12}$ Recently, our quantitative proteomics analysis identified multiple genes/pathways potentially associated with chemoresistance to MTX in JEG3 choriocarcinoma cells, which still awaits functional validation. ${ }^{13}$

Signaling Lymphocytic Activation Molecule Family Member 1 (SLAMF1, CD150) belongs to signaling lymphocyte activation molecule (SLAM) family of cell-surface receptors in hematopoietic cells. ${ }^{14}$ In hematologic malignancies, SLAMF1 expression is mainly expressed in cutaneous T-cell lymphomas, Hodgkin's lymphoma, chronic lymphocytic leukemia, and multiple myeloma. ${ }^{15-17}$ In chronic lymphocytic leukemia and Hodgkin's lymphoma, SLAMF1 plays an critical role in malignant cell fate decision and formation of tumor microenvironment. ${ }^{18,19}$ Recently, SLAMF1 has been shown to exert a crucial role in autophagy in chronic lymphocytic leukemia cells. ${ }^{20}$ However, the function of SLAMF1 in the regulation of MTX resistance still remains largely unknown. In our previous proteomics study, highly elevated expression of SLAMF1 was observed in MTX-resistant JEG3/MTX subline compared to parental JEG3, ${ }^{13}$ which sparked our interest to investigate its function in the chemoresistance to MTX in choriocarcinoma.

\section{Materials and Methods}

\section{Reagents and Cell Lines}

Primary antibodies used in this study were provided by the following sources: SLAMF1 (PA5-96046), Invitrogen (Carlsbad, CA, USA); $\beta$-actin (\#58169), LC3B (\#2775), p62 (\#8025), and cleaved Caspase-3 (\#9664), Cell Signaling (Danvers, MA, USA). MTX, Monodansylcadaverine (MDC), Bafilomycin A1, 5-bromo-2'-deoxyuridine (BrdU), and 3-Methyladenine (3-MA) were provided by Sigma-Aldrich (St. Louis, MO, USA). Human choriocarcinoma cell lines JEG3 and JAR were provided by ATCC culture collection (Manassas, VA, USA). MTX-resistant JEG3/MTX and JAR/MTX sublines were established as we described previously. ${ }^{13}$ The use of these choriocarcinoma cell lines in this study was approved by the institutional research ethics committee in Xiangya Hospital, Central South University. These cell lines were routinely grown in Dulbecco's Modified Eagle's medium (DMEM) supplemented with $10 \%$ fetal bovine serum. Lentiviral plasmids expressing non-targeting scramble or shSLAMF1 were provided by Genecopoeia Inc. (Rockville, MD, USA). Lentiviral plasmids LV105 expressing empty vector or SLAMF1 open reading frames (ORFs) were also provided by Genecopoeia Inc. The production of recombined lentivirus was performed according to the manufacturer's instruction. ${ }^{13}$

\section{Soft Agar Clonogenesis Assay}

Soft agar clonogenesis assay was used to examine the clonogenesis potential of choriocarcinoma cells as we described previously. ${ }^{21}$ After incubation with or without MTX for 10 12 days, the colonies ( $>50$ cells) were counted.

\section{Cell Viability Analysis}

Cell viability was evaluated with $\mathrm{CCK}-8$ assay as described previously. ${ }^{13}$ Briefly, choriocarcinoma cells were seeded in a 96 -well plate in triplicate $\left(3 \times 10^{3}\right.$ cells/ well). Cell viability was measured at 48 hours after incubation with MTX. $\mathrm{IC}_{50}$ values were calculated in SPSS software as we described previously. ${ }^{13}$

\section{BrdU Incorporation Assay}

BrdU incorporation assay was conducted using Cell Proliferation Assay Kit (Cell Signaling, Boston, MA) as we described previously. ${ }^{13}$ Briefly, choriocarcinoma cells were plated $\left(3 \times 10^{3}\right.$ cells/well) into a 96 -well plate overnight. After MTX incubation for 48 hours, choriocarcinoma cells were incubated with $\mathrm{BrdU}(10 \mu \mathrm{M}, 2$ hours). Incorporated $\mathrm{BrdU}$ was measured at $\mathrm{OD}_{450}$ using a Multiskan MK3 microplate reader.

\section{Protein Extraction and Western Blotting}

Western blotting was conducted as we described previously. ${ }^{13}$ Briefly, Cells were washed in cold PBS and lysed in a radioimmunoprecipitation assay (RIPA) buffer containing $50 \mathrm{mM}$ Tris-HCl, pH 7.4, $150 \mathrm{mM}$ $\mathrm{NaCl}, 1 \mathrm{mM}$ Phenylmethanesulfonyl fluoride (PMSF), 1 
$\mathrm{mM}$ Ethylenediamine tetra-acetic acid (EDTA), $5 \mathrm{mg} /$ $\mathrm{mL}$ aprotinin, $5 \mathrm{mg} / \mathrm{mL}$ leupeptin, $1 \%$ Triton $\mathrm{X}-100,1 \%$ sodium deoxycholate, and $0.1 \%$ sodium dodecyl sulfate (SDS). The cell extract with $25 \mu \mathrm{g}$ proteins was loaded on a $10 \%$ or $15 \%$ SDS-polyacrylamide gel and then transferred to polyvinylidene fluoride (PVDF) membranes. The membrane was incubated for $1 \mathrm{~h}$ in Trisbuffered saline (TBS) containing 5\% skim milk. After blotting with primary antibody, the protein expression was revealed with the appropriate horseradish peroxidase-labeled secondary antibody, which was detected using ECL chemiluminescence. Mouse anti-human $\beta$ Actin was used as loading control.

\section{Incorporation Assay}

MDC incorporation assay was conducted to measure the level of intracellular autophagy as described previously. ${ }^{22}$ Choriocarcinoma cells were incubated with $50 \mu \mathrm{M}$ MDC for $10 \mathrm{~min}$. The incorporated MDC was detected (excitation $380 \mathrm{~nm}$; emission 525nm) in F97Pro fluorospectrometer (Lengguang Technology, Shanghai, China).

\section{Transmission Electron Microscopy}

To demonstrate the autophagic morphology in choriocarcinoma cells, we performed transmission electron microscopy as described previously. ${ }^{22}$ JEG3 and JEG3/MTX cells were fixed with ice-cold glutaraldehyde ( $2 \%$ in $0.1 \mathrm{M}$ cacodylate buffer, $\mathrm{pH}$ 7.4) for $30 \mathrm{~min}$. After fixation, the samples were postfixed in $1 \% \mathrm{OsO} 4$ in the same buffer for $1 \mathrm{~h}$ and then subjected to the electron microscopic analysis. Representative areas were chosen for ultrathin sectioning and viewed with a Philips CM120 transmission electron microscope.

\section{Immunofluorescence Staining}

JEG3 and JEG3/MTX cells were seeded on the coverslips and grown overnight. Cells were fixed in $4 \%$ paraformaldehyde, permeated with $0.25 \%$ Triton X-100, blocked with $3 \%$ normal goat serum, stained with LC3 antibody overnight, and labeled with goat anti-rabbit IgG conjugated with tetramethylrhodamine (TRITC) (Zymed Laboratories, CA, USA). The cells were counterstained with sealant containing 40.6-diamidino2-phenylindole (DAPI) (Meilunbio, Dalian, China) and examined under an Olympus BX40 fluorescence microscope (Olympus, Japan).

\section{Statistical Analysis}

SPSS 16.0 was used to conduct the statistical analysis. Data were expressed as mean \pm standard deviation.
Student's $t$ test was conducted to compare the means of two groups. One-way ANOVA analysis was used to compare the means of three or more groups. $\mathrm{P}<0.05$ was considered statistically significant for all the statistical tests.

\section{Results \\ SLAMFI Expression is Increased in MTX-Resistant Cell Models}

In our previous proteomics study, ${ }^{13}$ we identified multiple genes/pathways potentially involved in MTX resistance in JEG3:JEG3/MTX cell models. SLAMF1 was shown to be the top protein highly enriched in JEG3/MTX subline ( $\mathrm{P}<0.05$; Figure 1A and B). Consistently, Western blotting analysis revealed an increase in SLAMF1 expression in MTX-resistant sublines compared to parental JEG3 and JAR cells, respectively (Figure 1C).

\section{Knockdown of SLAMFI Sensitized MTX-Resistant Choriocarcinoma Cells to MTX Treatment}

To examine the effect of SLAMF1 on the drug response of JEG3/MTX and JAR/MTX cells to MTX, we depleted SLAMF1 expression through shRNAmediated knockdown. The expression of SLAMF1 was mostly reduced by shRNA targeting SLAMF1 compared to scramble (Scr) control (Figure 2A). Knockdown of SLAMF1 enhanced MTX chemosensitivity, as $\mathrm{IC}_{50}$ value was lower in shSLAMF1 group than in Scr control in MTX-resistant JEG3 and JAR sublines (Figure 2B). In addition, the effect of SLAMF1 on cellular proliferative potential was examined by BrdU incorporation assay in JEG3/MTX and JAR/MTX cells. The shSLAMF1 group was found less BrdU incorporation compared with Scr group after incubation with MTX $(10 \mu \mathrm{M})$ for 48 hours (Figure 2C). Consistently, knockdown of SLAMF1 reduced soft agar clonogenesis in JEG3/MTX and JAR/MTX cells after MTX treatment $(10 \mu \mathrm{M})$, as compared with Scr control (Figure 2D).

\section{Over-Expression of SLAMFI Promotes Resistance to MTX in Choriocarcinoma Cells}

We next overexpressed SLAMF1 in order to examine its function in JEG3 and JAR cells. SLAMF1 


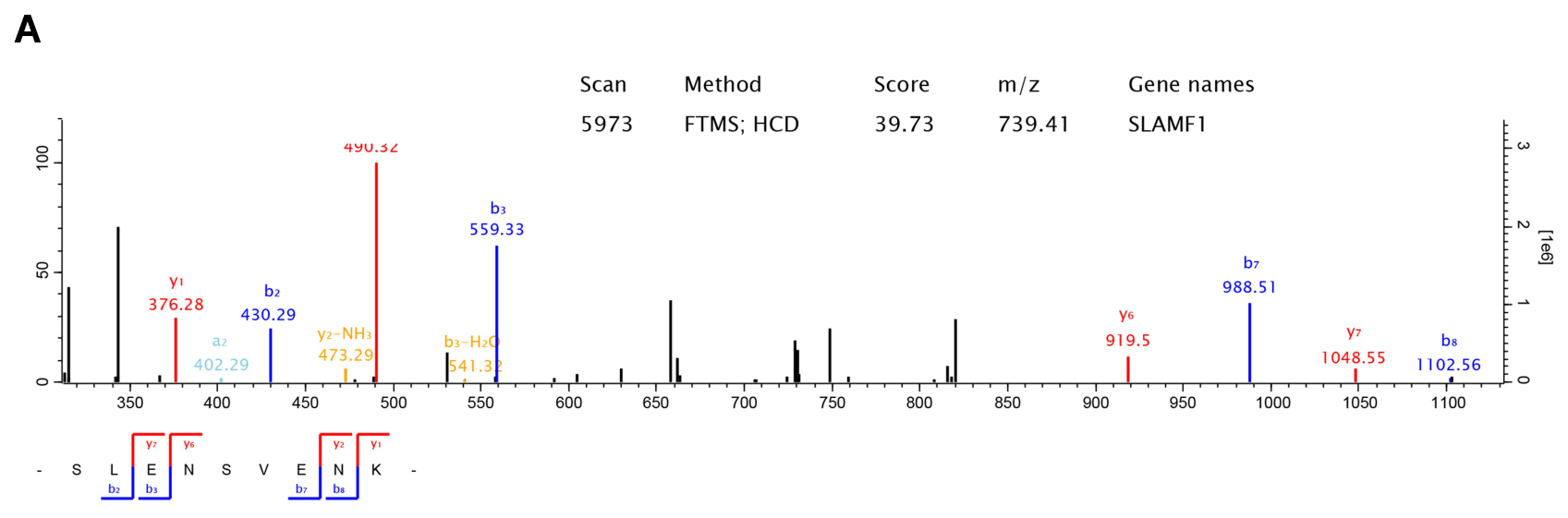

B



C



Figure I SLAMFI expression is increased in MTX-resistant choriocarcinoma cells. (A). The mass spectrum of SLAMFI unique peptide (SLENSVENK) identified by TMT labeling and LC-MS/MS. (B). Relative expression of SLAMFI protein in JEG3 and its MTX-resistant subline JEG3/MTX. *P<0.05. (C). Western blotting validation on SLAMFI expression in MTX-resistant choriocarcinoma sublines and their parental cell lines. $\beta$-actin was used as loading control.

expression was apparently increased in this SLAMF1 group compared to empty vector (EV) control (Figure 3A). Overexpression of SLAMF1 enhanced chemoresistance to MTX, as $\mathrm{IC}_{50}$ value was higher in SLAMF1 group than in EV control in JEG3 and JAR cells (Figure 3B). In addition, the effect of SLAMF1 on cellular proliferative potential was examined by BrdU incorporation assay in JEG3 and JAR cells. The SLAMF1 group was found more BrdU incorporation compared with EV group after incubation with MTX (3 $\mu \mathrm{M}$ ) for 48 hours (Figure 3C). Consistently, overexpression of SLAMF1 increased soft agar clonogenesis in JEG3 and JAR cells after MTX treatment ( $3 \mu \mathrm{M})$, as compared with EV control (Figure 3D).

\section{Autophagy Might Be Involved in MTX Resistance in Choriocarcinoma Cells}

SLAMF1 is a crucial regulator of autophagy in chronic lymphocytic leukemia cells, ${ }^{20}$ which might implicate autophagy as possible mechanism of chemoresistance to MTX in choriocarcinoma. As shown in Figure 4A, increased protein levels of autophagy-related genes LC3-II and p62 were observed in MTX-resistant sublines compared to parental choriocarcinoma cells, respectively. Consistently, More MDC incorporation was noted in MTX-resistant sublines than that in parental choriocarcinoma cells (Figure 4B). Electron microscopic observation on the ultrastructural features revealed enormous autophagic vacuoles in JEG3/MTX cells compared to JEG3 (Figure 4C). Consistently, punctate LC3 immunostaining was seen in JEG3/ MTX, which represents typical autophagy morphology (Figure 4D). Cell viability in MTX-resistant sublines was greatly reduced in MTX plus 3-MA group or MTX plus bafilomycin, group, as compared with either drug alone (Figure 4E). These results suggested that autophagy might serve as a prosurvival mechanism in MTXresistant choriocarcinoma cells. 
A

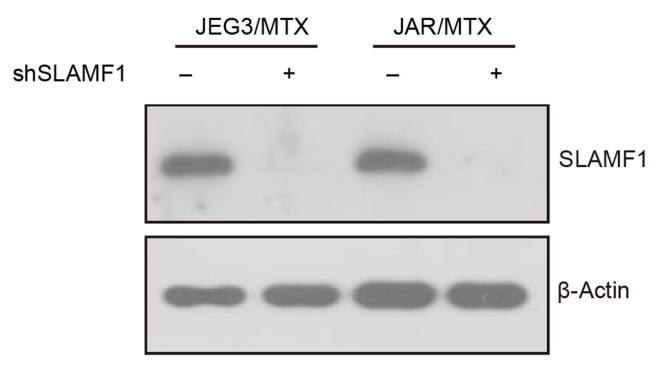

C

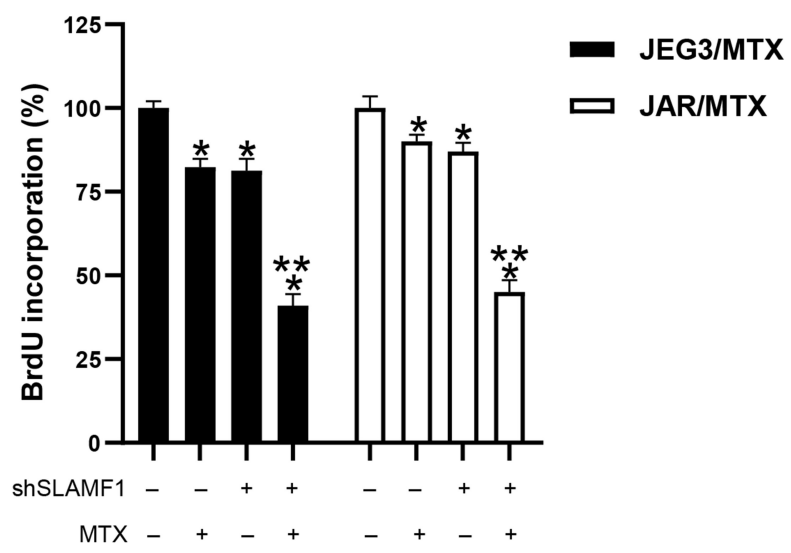

B



D

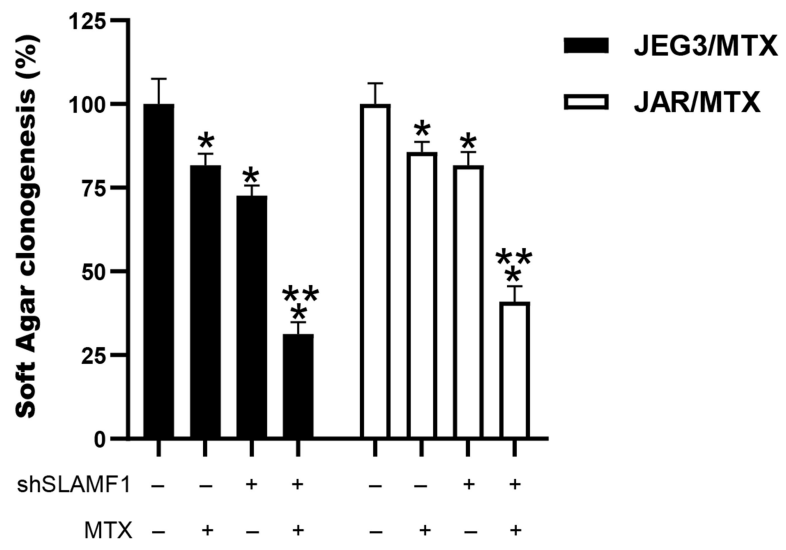

Figure 2 Knockdown of SLAMFI enhanced MTX chemosensitivity in MTX-resistant choriocarcinoma cell lines. (A). SLAMFI expression was markedly attenuated by genespecific shRNA in JEG3/MTX and JAR/MTX cells. (B). Knockdown of SLAMFI increased MTX sensitivity in JEG3/MTX and JAR/MTX cells. The cell viability in Scr or shSLAMFI without MTX treatment was regarded as $100 \%$, respectively. $n=3,{ }^{*} P<0.01$. (C). Knockdown of SLAMFI attenuated the proliferative potential of JEG3/MTX and JAR/MTX cells after MTX treatment for 48 hours. The BrdU incorporation in Scr without MTX treatment was regarded as $100 \%$. $\mathrm{n}=3$, $* \mathrm{P}<0.05$, as compared with Scr without MTX treatment. **P<0.05, as compared with Scr with MTX treatment. (D). Knockdown of SLAMFI impaired soft agar clonogenesis after MTX treatment in JEG3/ MTX and JAR/MTX cells. The colony formation in Scr group without MTX treatment was regarded as $100 \%$, respectively. $n=3$, *P<0.05, as compared with Scr without MTX treatment. $* * \mathrm{P}<0.05$, as compared with Scr with MTX treatment.

\section{SLAMFI Regulates Autophagy in Choriocarcinoma Cell Lines}

We next examined the effect of SLAMF1 on autophagy level in choriocarcinoma cells. Knockdown of SLAMF1 attenuated the expression of autophagy-related genes (LC3II and p62) and inhibited MDC incorporation compared to Scr control in JEG3/MTX and JAR/MTX cells (Figure 5A and B). In contrast, over-expression of SLAMF1 induced the expression of autophagy-related genes (LC3-II and p62) and induced MDC incorporation compared to empty vector (EV) control in JAR and JEG3 cells (Figure 5C and D).

\section{Depletion of SLAMFI Induced Apoptosis in JEG3/MTX and JAR/MTX Cells After MTX Treatment}

We depleted SLAMF1 expression in MTX-resistant choriocarcinoma cells in order to elucidate the role of autophagy on cellular apoptosis. Western blotting showed that SLAMF1 depletion could considerably reduce LC3-II and p62 levels in JEG3/MTX (Figure 6). Meanwhile, the level of apoptosis-related cleaved caspase-3 was significantly increased after MTX treatment in shSLAMF1 group compared to Scr control (Figure 6).

\section{Discussion}

The acquisition of chemoresistance to methotrexate (MTX) still remains one of the major challenges for choriocarcinoma treatment. Exploring the mechanism of MTX resistance would help to develop therapeutic strategy to combat MTX-resistant choriocarcinoma. The signaling lymphocytic activation molecule (SLAM) family has been shown to regulate inflammation and immune response. ${ }^{23}$ Recently, SLAM genes have been implicated in drug response regulation in cancerous cells. Fouquet 
A



C
B

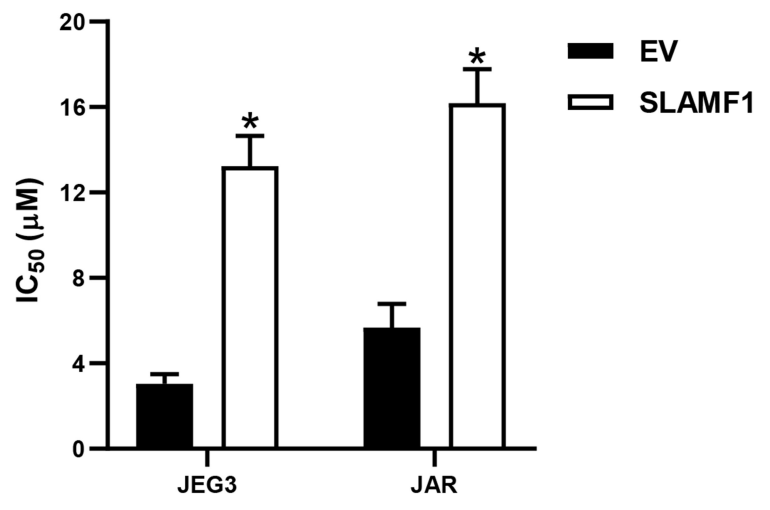



D

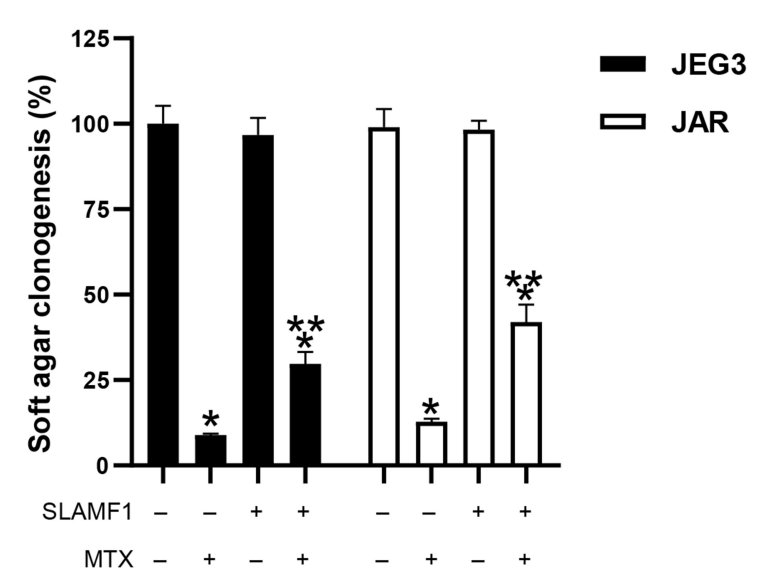

Figure 3 Over-expression of SLAMFI promoted chemoresistance to MTX in JEG3 and JAR cells. (A). SLAMFI expression was considerably increased in JEG3 and JAR cells. (B). Over-expression of SLAMFI promoted chemoresistance to MTX in JEG3 and JAR cells. $n=3$, *P<0.05. (C). Over-expression of SLAMFI enhanced the proliferative potential of choriocarcinoma cells after MTX treatment. The BrdU incorporation in EV of each cell line without MTX treatment was regarded as $100 \%$, respectively. $n=3$, *P $<0.05$, as compared with EV without MTX treatment; ${ }^{* *} \mathrm{P}<0.05$, as compared with EV with MTX treatment. (D). Over-expression of SLAMFI rescued soft agar clonogenesis after MTX treatment in choriocarcinoma cell lines. The colony formation in EV of each cell line without MTX treatment was regarded as I00\%, respectively. $\mathrm{n}=3$, ${ }^{*} \mathrm{P}<0.05$, as compared with EV without MTX treatment; ${ }^{*} \mathrm{P}<0.05$, as compared with EV with MTX treatment.

et al showed that SLAMF3 inhibits liver cancer growth and might predict resistance to sorafenib. ${ }^{24}$ Bologna et al showed that loss of SLAMF1 was associated with cellular response to fludarabine and ABT-737 in chronic lymphocytic leukemia cells. ${ }^{20}$ Recently, our proteomic profiling identified SLAMF1 as a potential regulator of MTX resistance in JEG3/JEG3/MTX cell models. ${ }^{13}$ Knockdown of SLAMF1 markedly attenuated cell viability and soft agar clonogenesis after incubation with MTX in JEG3/MTX and JAR/MTX cells. In contrast, overexpression of SLAMF1 rescued cell survival soft agar clonogenesis in JEG3 and JAR cells treated with MTX. Therefore, our findings might connect the novel function of the SLAMF1 with the modulation of drug resistance in choriocarcinoma.
Autophagy is an important cellular mechanism that recycles cytoplasmic components into lysosome for bulk degradation. $^{25,26}$ Physiologically, autophagy pathway is dramatically induced in order to survive from nutrient starvation and stress. ${ }^{27,28}$ Autophagy could also be induced by cancer therapy, which contributes to cancer cell survival and the eventual recurrence of the disease. ${ }^{29,30}$ Autophagy machinery could be activated in several cell models in response to MTX treatment. Xu et al showed that autophagy induction contributes to the resistance to methotrexate treatment in rheumatoid arthritis fibroblast-like synovial cells. ${ }^{31}$ Shen et al revealed a survival mechanism of the switch from ER stressinduced apoptosis to autophagy via ROS-mediated JNK/ p62 signals in methotrexate-resistant choriocarcinoma 
A

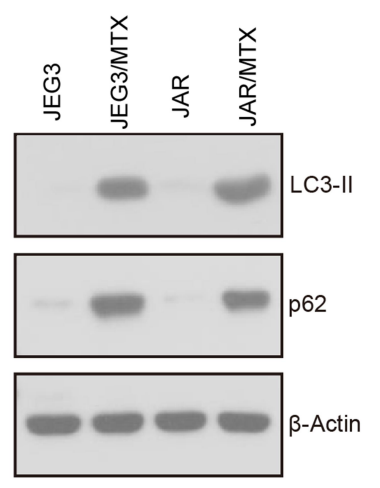

B

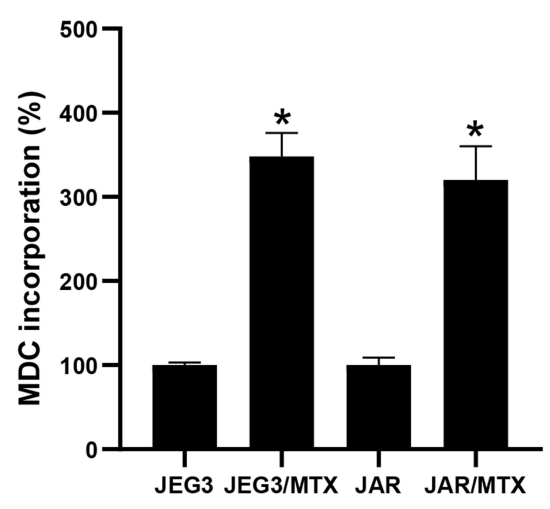

D
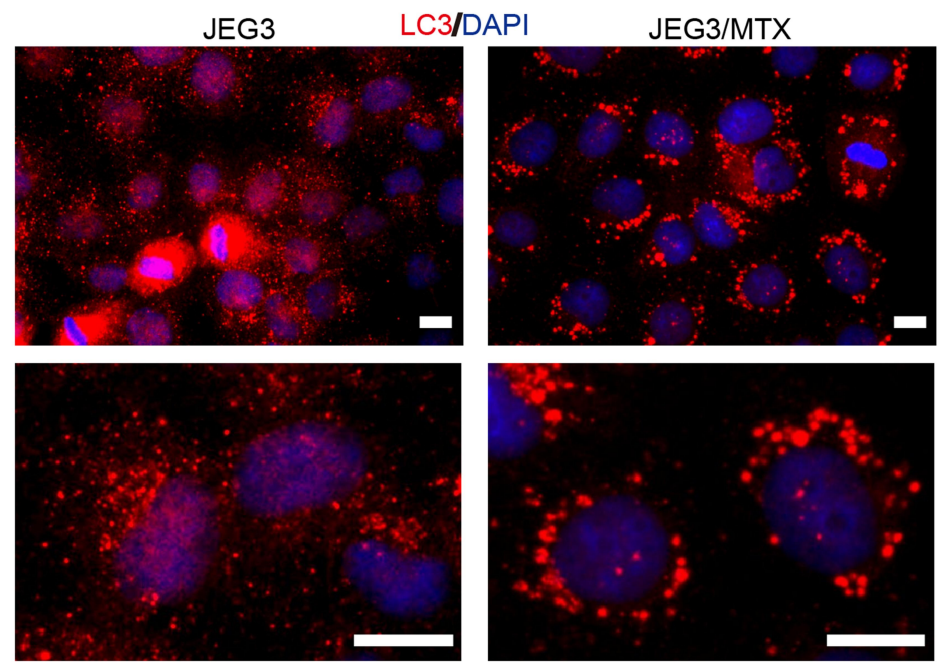

C

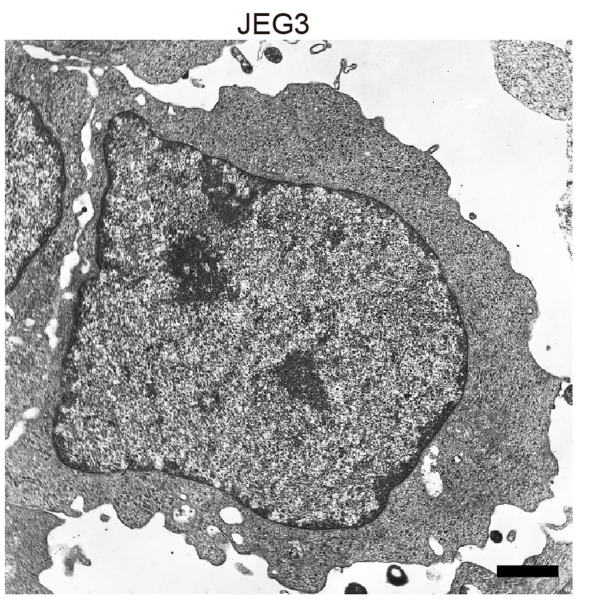

JEG3/MTX

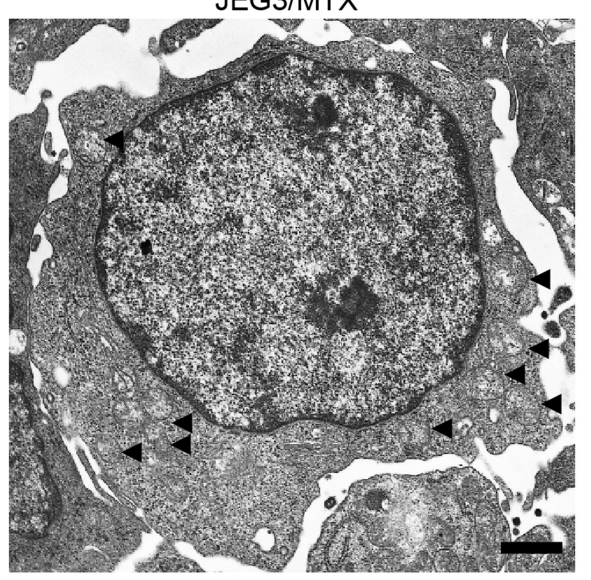

E


Figure 4 Autophagy might be involved in MTX resistance in MTX-resistant choriocarcinoma cells. (A). Western blotting analysis on the expression of autophagy-related genes LC3B and p62 in MTX-resistant choriocarcinoma sublines and parental cell lines. (B). MDC incorporation in MTX-resistant choriocarcinoma sublines and parental cell lines. $n=3, * P<0.05$. (C). Electron microscopic observation on the ultrastructural features of autophagy in JEG3 and JEG3/MTX cells. Black triangles indicated the autophagic vacuoles in JEG3/MTX cells. Bars: I $\mu$ m. (D). Punctate LC3 immunostaining was seen in JEG3/MTX compared to JEG3, which represents typical autophagy morphology. Bars: $10 \mu \mathrm{m}$. (E). Autophagy inhibitor 3-MA and bafilomycin Al enhanced MTX-induced cytotoxicity in MTX-resistant choriocarcinoma cells. $\mathrm{n}=3$, $* \mathrm{P}<0.05$, as compared with vehicle control. ${ }^{* *} \mathrm{P}<0.05$, as compared with either drug alone. 
A



C

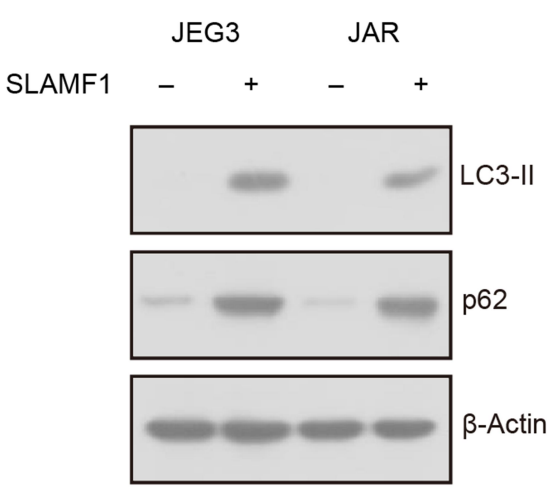

B
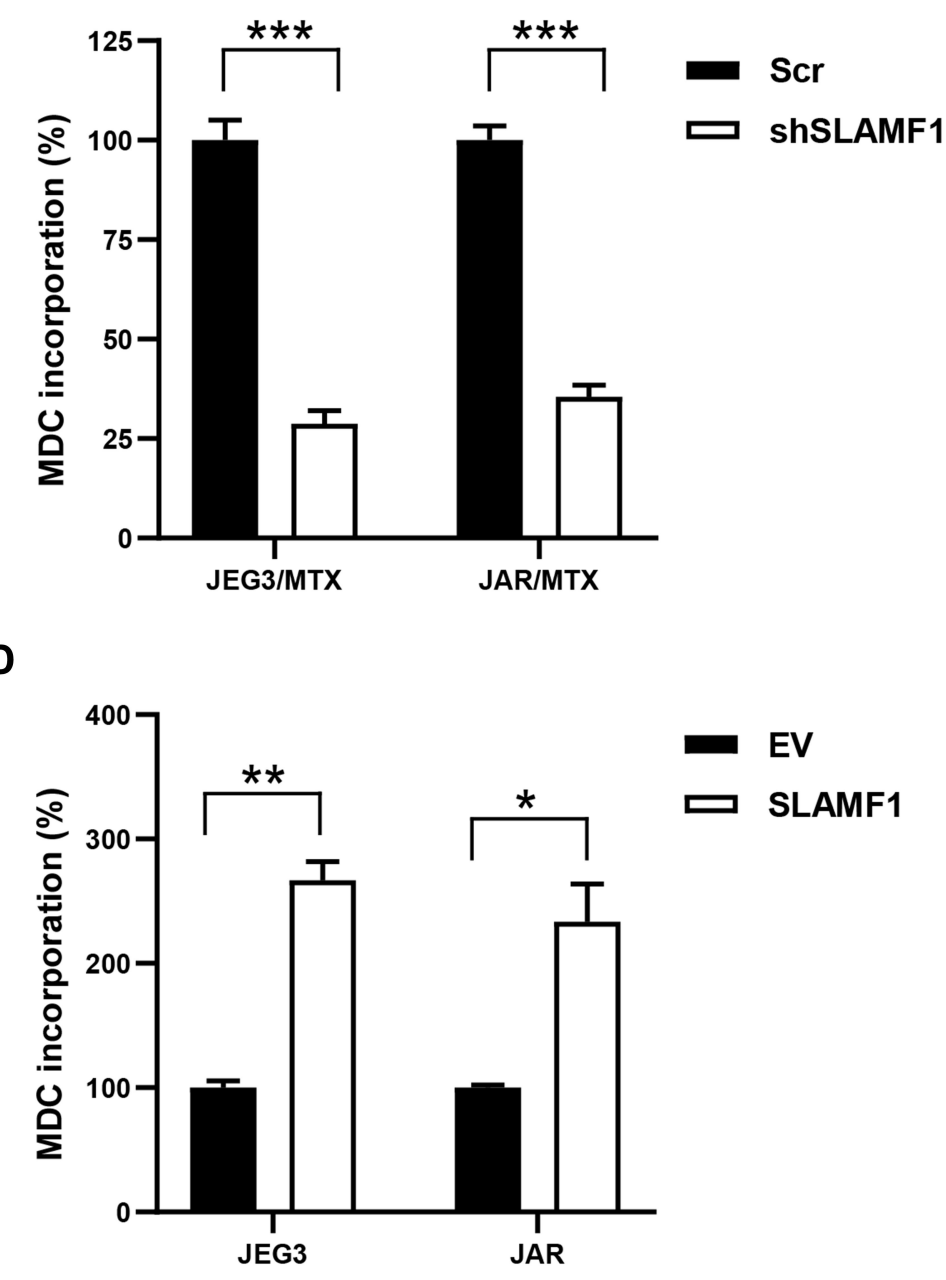

Figure 5 SLAMFI regulates autophagy in choriocarcinoma cells. (A). Knockdown of SLAMFI attenuated the expression of autophagy-related genes (LC3-II and p62) in MTX-resistant choriocarcinoma sublines. (B). MDC incorporation in MTX-resistant choriocarcinoma sublines with or without SLAMFI depletion. $\mathrm{n}=3$, $* * * \mathrm{P}<0.00 \mathrm{I}$. (C). SLAMFI over-expression increased the expression of autophagy-related genes (LC3-II and p62) in choriocarcinoma cells. $\beta$-actin was used as loading control. (D). MDC incorporation in choriocarcinoma cell lines with or without SLAMFI expression. $n=3, * P<0.05 ; * * P<0.01$.

cells. $^{32}$ Similarly, our findings showed that autophagyrelated genes (LC3-II, p62) were apparently increased in MTX-resistant choriocarcinoma cells compared to parental cell lines. Further, autophagy inhibitor 3-MA and Bafilomycin could enhance MTX-induced cytotoxicity in MTX-resistant choriocarcinoma cells. These results suggested that autophagy-related mechanisms might play an important role in MTX resistance in choriocarcinoma cells.

Recently, SLAMF1 has been shown to be a critical regulator of autophagy induced by fludarabine or ABT-737 in chronic lymphocytic leukemia cells, which might connect SLAMF1-regulated autophagy with drug response in cancer cells. ${ }^{20}$ However, the role of SLAMF1 in regulating MTX resistance in choriocarcinoma cells still remains unknown. Herein, we showed that manipulation of SLAMF1 expression could modulate autophagy in choriocarcinoma cell models, suggesting SLAMF1 might be a critical regulator of autophagy in choriocarcinoma cells. Further, autophagy regulated by SLAMF1 might be an important prosurvival mechanism, as knockdown of SLAMF1 suppressed autophagy and induced apoptosis in JEG3/MTX and JAR/MTX cells treated with MTX. Ma et al showed that SLAMF1 could recruit a Beclin-1/Vps34/UVRAG complex under oxidative stress condition, which leads to activation of the membrane NADPH oxidase 2 complexes. $^{33}$ Therefore, SLAMF1 might regulate autophagy-related pathway to activate antioxidant enzymes, which would be crucial in mediating chemoresistance to MTX in choriocarcinoma cells.

In conclusion, our findings suggest that SLAMF1 might promote MTX resistance via activating protective 



Figure 6 Depletion of SLAMFI induced apoptosis in MTX-resistant choriocarcinoma cells treated with MTX. Western blotting analysis detects the expression of LC3-II, p62 and cleaved caspase-3 after MTX treatment in shSLAMFI or Scr group.

autophagy in choriocarcinoma cell lines. Targeting SLAMF1 might be a useful therapeutic strategy to sensitize choriocarcinoma cells to MTX-based regimens.

\section{Acknowledgments}

This work was funded by the Natural Science Foundation of Hunan Province, China (2019JJ40492).

\section{Disclosure}

The authors have no competing interests to declare.

\section{References}

1. Cheung AN, Zhang HJ, Xue WC, Siu MK. Pathogenesis of choriocarcinoma: clinical, genetic and stem cell perspectives. Future Oncol. 2009;5(2):217-231. doi:10.2217/14796694.5.2.217

2. May T, Goldstein DP, Berkowitz RS. Current chemotherapeutic management of patients with gestational trophoblastic neoplasia. Chemother Res Practice. 2011;2011:806256. doi:10.1155/2011/806256

3. Matsui H, Suzuka K, Yamazawa K, et al. Relapse rate of patients with low-risk gestational trophoblastic tumor initially treated with single-agent chemotherapy. Gynecol Oncol. 2005;96(3):616-620. doi:10.1016/j.ygyno.2004.11.011

4. Maestá I, Nitecki R, Horowitz NS, Goldstein DP. Effectiveness and toxicity of first-line methotrexate chemotherapy in low-risk postmolar gestational trophoblastic neoplasia: the New England Trophoblastic Disease Center experience. Gynecol Oncol. 2018;148(1):161-167. doi:10.1016/j.ygyno.2017.10.028

5. Bolis G, Bonazzi C, Landoni $\mathrm{F}$, et al. EMA/CO regimen in high-risk gestational trophoblastic tumor (GTT). Gynecol Oncol. 1988;31 (3):439-444. doi:10.1016/S0090-8258(88)80029-5

6. Bower M, Newlands ES, Holden L, et al. EMA/CO for high-risk gestational trophoblastic tumors: results from a cohort of 272 patients. J Clin Oncol. 1997;15(7):2636-2643. doi:10.1200/ JCO.1997.15.7.2636
7. Wu J, Wang D. CLIC1 induces drug resistance in human choriocarcinoma through Positive regulation of MRP1. Oncol Res. 2017;25 (6):863-871. doi:10.3727/096504016X14772315906527

8. Ding R, Shi J, Pabon K, Scotto KW. Xanthines down-regulate the drug transporter ABCG2 and reverse multidrug resistance. Mol Pharmacol. 2012;81(3):328-337. doi:10.1124/mol.111.075556

9. Han B, Xiang Y, Wang Y, Wang Z, Zhang H, Huang S. Dihydrofolate reductase transcript level is not suitable for methotrexate-resistance prediction in choriocarcinoma cell line. Int $J$ Gynecol Cancer. 2010;20(7):1259-1263. doi:10.1111/IGC.0b013e3181f05128

10. Wang X, Zong L, Wang W, Yang J, Xiang Y. CD105 overexpression mediates drug-resistance in choriocarcinoma cells through BMP9/Smad pathway. J Cancer. 2020;11(2):272-283. doi:10.7150/jca.34965

11. Elias KM, Harvey RA, Hasselblatt KT, Seckl MJ, Berkowitz RS. Type I interferons modulate methotrexate resistance in gestational trophoblastic neoplasia. Am J Reprod Immunol. 2017;77(6):6. doi:10.1111/aji.12666

12. Cai J, Peng T, Wang J, et al. Isolation, Culture and Identification of Choriocarcinoma Stem-Like Cells from the Human Choriocarcinoma Cell-Line JEG-3. Cell Physiol Biochem. 2016;39(4):1421-1432. doi:10.1159/000447845

13. Jun F, Peng Z, Zhang Y, Shi D. Quantitative proteomic analysis identifies novel regulators of methotrexate resistance in choriocarcinoma. Gynecol Oncol. 2020;157(1):268-279. doi:10.1016/j.ygyno.2020.01.013

14. Dragovich MA, Mor A. The SLAM family receptors: potential therapeutic targets for inflammatory and autoimmune diseases. Autoimmun Rev. 2018;17(7):674-682. doi:10.1016/j.autrev.2018.01.018

15. Gordiienko I, Shlapatska L, Kovalevska L, Sidorenko SP. SLAMF1/ CD150 in hematologic malignancies: silent marker or active player? Clin Immunol. 2019;204:14-22. doi:10.1016/j.clim.2018.10.015

16. Yurchenko MY, Kovalevska LM, Shlapatska LM, Berdova GG, Clark EA, Sidorenko SP. CD150 regulates JNK1/2 activation in normal and Hodgkin's lymphoma B cells. Immunol Cell Biol. 2010;88(5):565-574. doi:10.1038/icb.2010.14

17. Gordiienko I, Shlapatska L, Kholodniuk V, et al. The interplay of CD150 and CD180 receptor pathways contribute to the pathobiology of chronic lymphocytic leukemia B cells by selective inhibition of Akt and MAPK signaling. PLoS One. 2017;12(10):e0185940. doi:10.1371/journal.pone. 0185940 
18. Gordienko IM, Shlapatska LM, Kovalevska LM, Sidorenko SP. Differential expression of CD150/SLAMF1 in normal and malignant B cells on the different stages of maturation. Exp Oncol. 2016;38 (2):101-107. doi:10.31768/2312-8852.2016.38(2):101-107

19. Rigolin GM, Saccenti E, Melandri A, et al. In chronic lymphocytic leukaemia, SLAMF1 deregulation is associated with genomic complexity and independently predicts a worse outcome. Br J Haematol. 2020. doi:10.1111/bjh.16865

20. Bologna C, Buonincontri R, Serra S, et al. SLAMF1 regulation of chemotaxis and autophagy determines CLL patient response. J Clin Invest. 2016;126(1):181-194. doi:10.1172/JCI83013

21. Jun F, Peng Z, Zhang Y, Shi D. Quantitative Proteomic Profiling Identifies SOX8 as Novel Regulator of Drug Resistance in Gestational Trophoblastic Neoplasia. Front Oncol. 2020;10:557. doi:10.3389/fonc. 2020.00557

22. Fu J, Shao C-J, Chen F-R, Ng H-K, Chen Z-P. Autophagy induced by valproic acid is associated with oxidative stress in glioma cell lines. Neuro Oncol. 2010;12(4):328-340. doi:10.1093/neuonc/nop005

23. Veillette A. SLAM-family receptors: immune regulators with or without SAP-family adaptors. Cold Spring Harb Perspect Biol. 2010;2 (3):a002469. doi:10.1101/cshperspect.a002469

24. Fouquet G, Debuysscher V, Ouled-Haddou H, et al. Hepatocyte SLAMF3 reduced specifically the multidrugs resistance protein MRP-1 and increases HCC cells sensitization to anti-cancer drugs. Oncotarget. 2016;7(22):32493-32503. doi:10.18632/oncotarget.8679

25. Glick D, Barth S, Macleod KF. Autophagy: cellular and molecular mechanisms. J Pathol. 2010;221(1):3-12. doi:10.1002/path.2697

26. Mizushima N, Komatsu M. Autophagy: renovation of cells and tissues. Cell. 2011;147(4):728-741. doi:10.1016/j.cell.2011.10.026
27. Ravanan P, Srikumar IF, Talwar P. Autophagy: the spotlight for cellular stress responses. Life Sci. 2017;188(188):53-67. doi:10.1016/j.1fs.2017.08.029

28. Puente C, Hendrickson RC, Jiang X. Nutrient-regulated Phosphorylation of ATG13 Inhibits Starvation-induced Autophagy. J Biol Chem. 2016;291(11):6026-6035. doi:10.1074/jbc. M115.689646

29. Sui X, Chen R, Wang Z, et al. Autophagy and chemotherapy resistance: a promising therapeutic target for cancer treatment. Cell Death Dis. 2013;4(10):e838. doi:10.1038/cddis.2013.350

30. Li X, Zhou Y, Li Y, et al. Autophagy: A novel mechanism of chemoresistance in cancers. Biomed Pharmacother. 2019;119:109415. doi:10.1016/j.biopha.2019.109415

31. Xu K, Cai Y-S, Lu S-M, et al. Autophagy induction contributes to the resistance to methotrexate treatment in rheumatoid arthritis fibroblast-like synovial cells through high mobility group box chromosomal protein 1. Arthritis Res Ther. 2015;17(1):374. doi:10.1186/ s13075-015-0892-y

32. Shen Y, Yang J, Zhao J, Xiao C, Xu C, Xiang Y. The switch from ER stress-induced apoptosis to autophagy via ROS-mediated JNK/p62 signals: A survival mechanism in methotrexate-resistant choriocarcinoma cells. Exp Cell Res. 2015;334(2):207-218. doi:10.1016/j. yexcr.2015.04.010

33. Ma C, Wang N, Detre C, Wang G, O'Keeffe M, Terhorst C. Receptor signaling lymphocyte-activation molecule family 1 (Slamf1) regulates membrane fusion and NADPH oxidase 2 (NOX2) activity by recruiting a Beclin-1/Vps34/ultraviolet radiation resistance-associated gene (UVRAG) complex. J Biol Chem. 2012;287(22):18359-18365. doi:10.1074/jbc.M112.367060

\section{Publish your work in this journal}

Cancer Management and Research is an international, peer-reviewed open access journal focusing on cancer research and the optimal use of preventative and integrated treatment interventions to achieve improved outcomes, enhanced survival and quality of life for the cancer patient.
The manuscript management system is completely online and includes a very quick and fair peer-review system, which is all easy to use. Visit http://www.dovepress.com/testimonials.php to read real quotes from published authors. 